\title{
Erste Hilfe für die Seele
}

\author{
Erhard Taverna
}

Traumatische Ereignisse können bei Menschen körperliche und psychische Folgen haben. Um eine akute traumatische Reaktion oder mögliche spätere Fehlentwicklungen abzuwenden oder zu mildern, wurden vorbeugende Methoden wie die psychologische erste Hilfe entwickelt. In allen Kantonen bieten sogenannte "care teams» eine Notfallseelsorge in Krisensituationen an. Die Strukturen dieser Teams und die Selektion, Aus- und Weiterbildung der Laienhelfer und -helferinnen sind im Prinzip überall ähnlich.

\section{Die Arbeit ...}

Seit 1999 verfügen die Kantone Appenzell-Ausserrhoden und -Innerrhoden über ein Care team, das «sofortige, unmittelbare und zeitlich begrenzte Krisenbegleitung oder gezielte Verarbeitungshilfe (Debriefing)» anbietet. Die Bewältigung von belastenden Erfahrungen wurde inzwischen in etwa zwei Dritteln den Betroffenen und deren Angehörigen und in einem Drittel den Mitgliedern der Feuerwehr, Polizei und Rettungsdienste angeboten. Die Organisation vermittelt im Bedarfsfall weitergehende professionelle Therapien und übernimmt damit auch eine Triagefunktion. Wie in den Kantonen Bern und Luzern ist das Team dem Zivilschutz eingegliedert, was den Vorteil hat, dass der Bund für den Erwerbsausfall aufkommt, während die Kantone die Ausbildung finanzieren. Konzeptionell sind die Direktionen der Sicherheit und Gesundheit für das Einsatzteam, deren fachtechnische Berater und die Leitung zuständig. Betroffene oder Helfer können über die kantonale Notrufzentrale via Einsatzleitung ein Aufgebot auslösen. Meistens ist es die Polizei, die vor Ort erst einmal die Situation abklärt, bevor sie über dieses Hilfsangebot informiert oder gegebenenfalls direkt ein Aufgebot auslöst. Wer sich für diese Aufgabe interessiert, muss erst einmal einen Fragebogen und vor allem ein Aufnahmegespräch hinter sich bringen. Das multimodale Interview versucht mit Hilfe eines Kompetenzeninventars, die Spreu vom Weizen zu trennen, denn Engagement und Initiative allein ersetzen nicht die geforderte Belastbarkeit, das nötige Einfühlungsvermögen und die emotionale Stabilität. Ein 2tägiger Einführungskurs bei Frau Dr. med. Gisela Perren im Institut Psychotrauma in Visp macht den Anfang, gefolgt von einem 2tägigen Fortbildungskurs, ebenso langen Auffrischungskursen und jährlichen 3-4 internen Weiterbildungskursen mit Spezialisten und Partnerorganisationen. In 23 Einsätzen wurden seit 200164 Helfer für ein Mehrfaches an Betroffenen angefordert; am häufigsten bei Verkehrsunfällen, Bränden, Arbeitsunfällen mit Todesfolgen und Suiziden.

\section{... und die Folgen}

Ein Merkblatt des Bundesamtes für Zivilschutz definiert die psychologische erste Hilfe als «den angemessenen Umgang mit Personen in einer akuten psychischen (seelischen) Notfallsituation», ausgelöst durch belastende Faktoren wie Schmerz, Angst, Kontrollverlust und Fremdbestimmung. Zu den Grundsätzen der ersten Hilfe gehören die Betreuung vor Ort, so rasch wie möglich und nach einfachen Methoden (keine Psychotherapie). Der Helfer soll mit den typischen Emotionen von Betroffenen wie Ungewissheit, Desorientierung, Schock, Leugnung, Misstrauen, Wut, Schuldgefühle, Trauer und Selbstaggressionen umgehen können.

Die psychologische erste Hilfe wurde bei uns erstmals in Armeereglementen als psychologische Kameradenhilfe eingeführt. Das zunehmenden fachliche Interesse für posttraumatische Belastungsstörungen (PTBS) und die Popularisierung durch die Medien verhalfen der Krisenintervention zur breiten Akzeptanz in der Bevölkerung und bei Politikern. Bei privaten und öffentlichen Unternehmen gibt es auch ökonomische Motive, weil die Erfahrungen zeigen, dass eine gute Betreuung auch die Prozessfreudigkeit und die Ansprüche auf Schadenersatz dämpft. Der relativ grosse finanzielle Aufwand wird mit den vermiedenen Langzeitschäden gerechtfertigt. Ob dies tatsächlich der Fall ist, wird in vielen Studien kontrovers beurteilt. Amerikanische Prävalenzstudien zur Entwicklung einer PTBS zeigen diese eher als Ausnahme denn als Regel. Zwar waren rund $60 \%$ aller untersuchten Personen mindestens einmal im Leben einem traumatischen Erlebnis ausgesetzt, doch nur 5\% der Männer und 10\% der Frauen entwickelten psychische Spätschäden. Bei einem massiven Trauma entwickelte rund ein Viertel eine Störung, am häufigsten nach einer Vergewaltigung. 
Bei rund 90\% aller Patienten mit einer PTBS wurde eine Komorbidität mit einer weiteren psychiatrischen Störung, vor allem Sucht und Depression, festgestellt.

Das Angebot ist publikumswirksam und für die Betroffenen kostenlos. Wie überall im Gesundheitswesen stimuliert das Angebot die Nachfrage. Unterbelegte Sanatorien und Zivil- schutzämter mit schwindenden Kernaufgaben haben eines gemeinsam: sie suchen sich neue Betätigungsfelder. Entscheidend ist aber auch die Eintrittsschwelle. Im Nachbarkanton St. Gallen werden die Helferinnen und Helfer nicht über die Notrufzentrale der kantonalen Polizei, sondern die Psychiatrischen Kliniken aufgeboten. Konsequenz: weit weniger Nachfragen!

\section{Ende des Projektes «Ein Tag Notfalldienst ...»}

Der Aufruf von Prof. Hans Stalder, dem Präsidenten der Kommission «Recherches et réalisations en médecine appliquée» (RRMA) der Schweizerischen Akademie der Medizinischen Wissenschaften (SAMW), über die Erfahrungen im Notfalldienst zu berichten, ist auf ein erfreuliches Echo gestossen. Über dreissig Beiträge sind eingetroffen; die meisten davon sind in der Zwischenzeit in der Schweizerischen Ärztezeitung (SÄZ) erschienen. Aus Reaktionen von Kolleginnen und Kollegen wissen wir, dass die Beiträge jeweils mit grossem Interesse gelesen werden. Die RRMA beendet nun dieses Projekt. Die noch vorliegenden Beiträge werden in dieser Nummer der SÄZ veröffentlicht. Eine Analyse der eingegangenen Beiträge aus berufener Hand wird die Serie beschliessen. Die SAMW dankt an dieser Stelle den Autorinnen und Autoren der Beiträge für ihr Engagement und ihre Bereitschaft, andere an ihren Erfahrungen teilhaben zu lassen.

\section{Fin du projet «Un jour de garde...»}

L'appel de M. le Professeur Hans Stalder, président de la commission «Recherches et réalisations en médecine appliquée» (RRMA) de l'Académie Suisse des Sciences Médicales (ASSM), de rapporter les expériences faites au service médical d'urgence a eu un écho très satisfaisant. Plus de trente contributions ont été soumises; la plupart d'entre elles est déjà parue dans le Bulletin des médecins suisses (BMS) et elles sont lues avec beaucoup d'intérêt, comme nous ont communiqué un bon nombre de collègues. La RRMA a mis un terme à ce projet. Les dernières contributions sont publiées dans l'édition actuelle du BMS et la série sera conclue d'une analyse d'expert. L'ASSM exprime ses remerciements aux auteurs des contributions pour leur engagement et leur volonté de partager leurs expériences à autrui. 